\title{
Treinamento de Caminhada Melhora a Variabilidade da Pressão Arterial Ambulatorial em Claudicantes
}

\author{
Walking Training Improves Ambulatory Blood Pressure Variability in Claudication \\ Marcel da Rocha Chehuen, ${ }^{10}$ Gabriel Grizzo Cucato, ${ }^{2}$ Celso Ricardo Fernandes de Carvalho, ${ }^{3}$ Antonio Eduardo \\ Zerati, ${ }^{3}$ Anthony Leicht, ${ }^{4}$ Nelson Wolosker, ${ }^{5}$ Raphael Mendes Ritti-Dias, ${ }^{(0)}$ Claudia Lucia de Moraes Forjaz ${ }^{10}$ \\ Universidade de São Paulo - Escola de Educação Física e Esporte, ${ }^{1}$ São Paulo, SP - Brasil \\ Northumbria University, Newcastle Upon Tyne, ${ }^{2}$ Reino Unido \\ Hospital das Clinicas (HCFMUSP), Faculdade de Medicina, Universidade de Sao Paulo, ${ }^{3}$ São Paulo, SP - Brasil \\ James Cook University, ${ }^{4}$ Queensland - Austrália \\ Hospital Israelita Albert Einstein, ${ }^{5}$ São Paulo, SP - Brasil \\ Universidade Nove de Julho - Programa de Pós-Graduação em Ciências da Reabilitação, ${ }^{6}$ São Paulo, SP - Brasil
}

\section{Resumo}

Fundamento: $O$ treinamento de caminhada (TC) melhora a capacidade de caminhar e reduz a pressão arterial (PA) clínica em pacientes com doença arterial periférica (DAP), mas seus efeitos na PA ambulatorial permanecem desconhecidos.

Objetivos: Investigar o efeito de 12 semanas de TC na PA ambulatorial e sua variabilidade em pacientes com DAP.

Métodos: Trinta e cinco pacientes do sexo masculino com DAP e sintomas de claudicação foram alocados aleatoriamente em dois grupos: controle $(n=16,30$ min de alongamento) e TC $(n=19,15$ séries de 2 minutos de caminhada na frequência cardíaca em que ocorreu limiar de dor intercalados por 2 minutos de repouso em pé). Antes e depois de 12 semanas, a PA ambulatorial de $\mathbf{2 4}$ horas foi avaliada. Os índices de variabilidade da PA ambulatorial avaliados em ambos os momentos incluíram o desvio-padrão de 24 horas $\left(\mathrm{DP}_{24}\right)$, o desvio-padrão ponderado de vigília e sono $\left(\mathrm{DP}_{\mathrm{vs}}\right)$ e a variabilidade real média de 24 horas $\left(\mathrm{VRM}_{24}\right)$. Os dados foram analisados por ANOVAs mistas de dois fatores, considerando significativo $\mathbf{P}<0,05$.

Resultados: Após 12 semanas, nenhum dos grupos apresentou alterações na PA de 24 horas, vigília e sono. O TC diminuiu as variabilidades da PA sistólica e média (PA sistólica - 13,3 $\pm 2,8$ vs 11,8 $\pm 2,3 ; 12,1 \pm 2,84$ vs 10,7 $\pm 2,5$; e 9,4 \pm 2,3 vs $8,8 \pm 2,2$ mmHg; PA média - 11,0 $\pm 1,7$ vs 10,4 $\pm 1,9 ; 10,1 \pm 1,6$ vs 9,1 $\pm 1,7 ;$ e 8,0 $\pm 1,7$ vs 7,2 $\pm 1,5$ mmHg para DP m $^{\prime}$

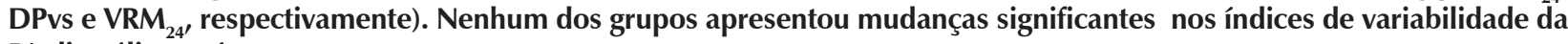
PA diastólica após 12 semanas.

Conclusão: O TC não altera os níveis ambulatoriais da PA, mas diminui a sua variabilidade em pacientes com DAP. Essa melhora pode ter um impacto favorável no risco cardiovascular de pacientes com DAP sintomática. (Arq Bras Cardiol. $2021 ; 116(5): 898-905)$

Palavras-chave: Claudicação Intermitente; Caminhada; Pressão Arterial; Monitoração Ambulatorial da Pressão Arterial; Fraqueza Muscular; Treinamento Aeróbico.

\footnotetext{
Abstract

Background: Walking training (WT) improves walking capacity and reduces clinic blood pressure (BP) in patients with peripheral artery disease (PAD), but its effects on ambulatory BP remains unknown.

Objectives: To investigate the effect of 12 weeks of WT on ambulatory BP and its variability in patients with PAD.

Methods: Thirty-five male patients with PAD and claudication symptoms were randomly allocated into two groups: control ( $n=16,30 \mathrm{~min}$ of stretching) and WT ( $n=19,15$ bouts of 2 min of walking at the heart rate of leg pain threshold interspersed by 2 min of upright rest). Before and after 12 weeks, 24-hour ambulatory BP was assessed. Ambulatory BP variability indices assessed at both time points included the 24-hour standard deviation $\left(S D_{24}\right)$, the awake and asleep weighted standard deviation $\left(S D_{d n}\right)$, and the 24-hour average real variability $\left(A R V_{24}\right)$. Data were analyzed by mixed two-way ANOVAs, considering $P<0.05$ as significant.
}

Correspondência: Cláudia Lúcia de Moraes Forjaz •

Universidade de São Paulo - Escola de Educação Física e Esporte,

Av. Prof. Mello Moraes, 65 - Cidade Universitária - CEP: 05508-030 - São Paulo - SP

E-mail: cforjaz@usp.br

Artigo recebido em 21/11/2019, revisado em 03/02/2020, aceito em 16/03/2020

DOI: https://doi.org/10.36660/abc.20190822 
Results: After 12 weeks, neither group had significant changes in 24-hour, awake and sleep BPs. The WT decreased systolic and mean BP variabilities (Systolic BP $-13.3 \pm 2.8$ vs 11.8 $\pm 2.3,12.1 \pm 2.84$ vs $10.7 \pm 2.5$ and $9.4 \pm 2.3$ vs $8.8 \pm 2.2 \mathrm{mmHg}$ ); Mean BP $-11.0 \pm 1.7$ vs $10.4 \pm 1.9$, $10.1 \pm 1.6$ vs $9.1 \pm 1.7$ and 8.0. \pm 1.7 vs $7.2 \pm 1.5 \mathrm{mmHg}$ for $S D_{24} S D_{d n}$ and $A R V_{24}$ respectively). Neither group had significant changes in diastolic BP variabilities after 12 weeks.

Conclusion: The WT does not change ambulatory BP levels but decreases ambulatory BP variability in patients with PAD. This improvement may have a favorable impact on the cardiovascular risk of patients with symptomatic PAD. (Arq Bras Cardiol. 2021; 116(5):898-905)

Keywords: Intermittent Claudication; Walking; Blood Pressure; Blood Pressure Monitoring Ambulatory; Muscle Weakness; Endurance Training.

Full texts in English - http://www.arquivosonline.com.br

\section{Introdução}

A claudicação intermitente, o sintoma mais prevalente da doença arterial periférica (DAP), prejudica a capacidade de locomoção, afetando os níveis de atividade física ${ }^{1}$ e a qualidade de vida do paciente. ${ }^{2}$ Além disso, essa limitação funcional está associada ao aumento das taxas de eventos cardiovasculares fatais e não fatais nessa população. ${ }^{3}$

Dentre as doenças cardiovasculares, a hipertensão arterial é uma comorbidade frequente, afetando mais de $80 \%$ dos pacientes com DAP, 4 Pacientes com DAP comumente apresentam valores de pressão arterial (PA) clínica e, principalmente, ambulatorial mais altos que indivíduos saudáveis. ${ }^{5}$ Recentemente a capacidade de caminhada foi negativamente associada com a PA ambulatorial na DAP, ${ }^{6}$ indicando um pior controle pressórico em pacientes com maior comprometimento funcional. Assim, estratégias terapêuticas que aumentem a capacidade funcional, como o treinamento de caminhada, podem melhorar os desfechos cardiovasculares e reduzir o risco cardiovascular nesse grupo.

Recentemente, demonstramos que o treinamento de caminhada (TC) supervisionado melhora a capacidade de caminhada e reduz a PA clínica de pacientes com DAP sintomática, ${ }^{7}$ porém seus efeitos na PA ambulatorial permanecem desconhecidos. Essa é uma questão importante, uma vez que a PA ambulatorial é um preditor mais forte de mortalidade por causas cardiovasculares do que a PA clínica. ${ }^{8}$ Além disso, um estudo anterior não relatou nenhum efeito do treinamento resistido de nos níveis ambulatoriais da PA, mas observou uma melhora na variabilidade da PA ambulatorial, que é um forte marcador de lesão de órgãos-alvo, eventos cardiovasculares e mortalidade. ${ }^{10}$ Uma vez que o treinamento aeróbico, como a caminhada, promove redução considerável nos níveis de PA ambulatorial em comparação ao treinamento resistido em populações normotensas e hipertensas, ${ }^{11}$ é possível supor que esta modalidade de exercício também possa melhorar a PA ambulatorial e sua variabilidade em pacientes com DAP, o que precisa ser verificado. Assim, o objetivo deste estudo foi investigar os efeitos do TC na PA ambulatorial e sua variabilidade em pacientes com DAP sintomática.

\section{Métodos}

\section{População do estudo}

Este é um dado complementar de um estudo anterior. ${ }^{7}$ Os pacientes foram recrutados no Ambulatorio de Cirurgia Vascular do Hospital das Clínicas da Universidade de São Paulo, Brasil. Foram convidados pacientes do sexo masculino com diagnóstico prévio de DAP e sintomas de claudicação intermitente. ${ }^{12}$ Os critérios de inclusão foram: (a) idade $\geq$ 50 anos; (b) índice tornozelo-braquial (ITB) $\leq 0,90^{11,12}$; c) DAP classe II segundo os critérios de Fontaine; ${ }^{13}$ (d) índice de massa corporal $<35 \mathrm{~kg} / \mathrm{m}^{2}$; (e) PA sistólica em repouso $<160$ $\mathrm{mmHg}$ e PA diastólica $<105 \mathrm{mmHg}$; (f) não estar tomando $\beta$-bloqueadores ou bloqueadores dos canais de cálcio não diidropiridínicos; (g) ausência de neuropatia autonômica cardiovascular nos pacientes diabéticos; ${ }^{14}$ (h) capacidade de caminhar por pelo menos 2 minutos a 3,2 km/h em uma esteira; (i) capacidade de realizar um teste incremental em esteira que seja limitado por por sintomas de claudicação intermitente; (j) ausência de isquemia miocárdica ou arritmias complexas durante um teste máximo em esteira; $(k)$ diminuição de pelo menos 15\% no ITB após um teste máximo em esteira; e (I) não estar envolvido em programa de exercícios físicos. Além disso, os pacientes não eram incluídos se tivessem pelo menos um dos seguintes critérios: 1) cirurgia de revascularização ou angioplastia há menos de um ano; 2) uso de vasodilatadores periféricos, 3) amputação de membros inferiores e 4) problemas ortopédicos que contraindicassem o TC. Os indivíduos foram excluídos quando seus medicamentos foram alterados durante o estudo. O protocolo do estudo foi registrado no Brazilian Clinical Trials (RBR-7M3D8W) e aprovado pelo Comitê de Ética em Pesquisa em Seres Humanos da Escola de Educação Física e Esporte da Universidade de São Paulo (processo: 392008/55) e do Hospital das Clínicas (processo: 1179/09), sendo conduzido de acordo com a Declaração de Helsinque. Um termo de consentimento livre e informado foi obtido de todos os pacientes antes da participação.

\section{Triagem dos participantes}

O diagnóstico de DAP foi feito com base no histórico clínico e na medida do ITB em repouso e após teste máximo em esteira. ${ }^{15}$ A PA sistólica do braço foi medida pelo método auscultatório e a PA sistólica do tornozelo de cada perna foi avaliada com um doppler vascular (Martec, DV 6000, Ribeirão Preto, Brasil). Para cada paciente, foi registrado o ITB de menor valor. A massa corporal e a altura foram medidas (Welmy, 110, São Paulo, Brasil), e o índice de massa corporal foi calculado. A PA braquial em repouso foi medida em duas visitas, sendo o valor médio calculado e utilizado na análise. Em cada visita, após cinco minutos em repouso sentado, foram realizadas três medidas auscultatórias da PA em cada braço, sendo registrado o maior valor médio. O uso de medicamentos e os hábitos de exercícios foram avaliados por meio de entrevista, Nos diabéticos, a presença de neuropatia autonômica cardiovascular foi avaliada de acordo com as 
recomendações da American Diabetes Association. ${ }^{14} \mathrm{O}$ tratamento medicamentoso foi mantido constante para todos os pacientes ao longo do estudo.

\section{Desenho do estudo}

O desenho experimental está descrito na Figura 1. O estudo foi composto por uma triagem inicial que incluiu um teste máximo em esteira utilizando o protocolo de Gardner para avaliar o limiar de dor. ${ }^{16} \mathrm{Em}$ seguida, os indivíduos que atenderam a todos os critérios do estudo realizaram uma monitorização ambulatorial da PA de 24 horas no início e 12 semanas após a intervenção. Os pacientes foram alocados de forma aleatória, por meio de um programa online específico (www.randomizer.org) em dois grupos: grupo treinamento de caminhada (GTC) e grupo controle (GC)

Para todas as avaliações, as recomendações prévias incluíam a não realização de exercício vigoroso nas 48 horas anteriores, realização de uma refeição leve 2 horas antes, a não ingestão de alimentos com propriedades estimulantes como cafeína, bebida alcoólica ou o consumo de tabaco nas 12 horas anteriores. As avaliações clínicas foram realizadas pela manhã em um laboratório com temperatura controlada $\left(20-22{ }^{\circ} \mathrm{C}\right)$

\section{Medidas}

\section{Desfecho primário: pressão arterial ambulatorial}

A monitorização ambulatorial da PA foi realizada com um aparelho oscilométrico não invasivo (SpaceLabs Medical Inc, 90207, Washington, EUA) posicionado no braço não dominante e programado para realizar medidas a cada 15 minutos durante 24 horas. A precisão do dispositivo foi confirmada por um esfigmomanômetro de mercúrio antes do uso.

Para a análise, os níveis ambulatoriais de PA sistólica, diastólica e média foram calculados pela média de todas as medidas realizadas por 24 horas, bem como durante os períodos de vigília e sono relatados pelo paciente. Além disso, a variabilidade da PA ambulatorial foi calculada para a PA sistólica, diastólica e média usando três índices diferentes: ${ }^{17}$ desvio-padrão de 24 horas $\left(\mathrm{DP}_{24}\right)$; desvio-padrão ponderado em vigília e durante o sono $\left(\mathrm{DP}_{\mathrm{vS}}\right)$ e a variabilidade real média de 24 horas $\left(\mathrm{VRM}_{24}\right)$. Esses índices foram calculados conforme relatado anteriormente. ${ }^{17}$ Resumidamente, o DP 24 foi calculado pelo desvio-padrão (DP) ao longo de 24 horas ponderado pelo intervalo de tempo entre as medidas. DPvs foi calculado pela média de DP de vigília e sono corrigido para o número de horas de cada um desses períodos [ou seja, DPvs $=[($ DP vigília $x$ horas de vigília $)+($ DP sono $x$ horas de sono)] / horas de vigília + horas de sono]. A VRM foi calculada pela média das diferenças absolutas entre as medições consecutivas, contabilizando a ordem de medição pela seguinte fórmula:

$$
V R M_{24}=\frac{1}{\Sigma_{w}} \sum_{k=1}^{n-1} w \times\left|P A_{k+1}-P A_{k}\right|
$$

Onde k varia de 1 a N-1, PA o valor da pressão arterial e w é o intervalo de tempo entre PAk e PAk+1. N é o número de registros válidos da PA.

\section{Intervenções}

Detalhes das intervenções foram relatados anteriormente. ${ }^{7}$ Resumidamente, as intervenções foram realizadas duas vezes por semana durante 12 semanas e supervisionadas por um dos pesquisadores. Os pacientes do GC realizaram exercícios de alongamento por 30 minutos. Os pacientes do GTC realizaram 15 séries de 2 minutos de caminhada em uma esteira, intercaladas por 2 minutos de repouso. Durante cada série de caminhada, a velocidade foi mantida em 3,2 km/h e a intensidade foi ajustada pela inclinação da esteira para manter a frequência cardíaca dentro de 4 bpm da frequência cardíaca obtida no limiar de dor avaliado durante o teste máximo em esteira $^{18}$ (por exemplo, se o paciente relatou o limiar de dor durante teste máximo em esteira a 100 bpm, a frequência cardíaca durante cada série era mantida entre 96 a 104 bpm).

\section{Análise estatística}

Conforme descrito anteriormente, ${ }^{7}$ o tamanho da amostra foi estimado considerando-se um poder de $90 \%$, erro alfa de $5 \%$ e desvio-padrão de $3 \mathrm{mmHg}$ para a PA sistólica. O tamanho mínimo necessário para detectar uma diferença de $4 \mathrm{mmHg}$ foi de 7 indivíduos em cada grupo.

A normalidade da distribuição dos dados e a homogeneidade da variância foram avaliadas pelos testes de Shapiro-Wilk e Levene, respectivamente. Distribuições que não atenderam aos critérios de normalidade foram normalizadas usando transformações logarítmicas. No início do estudo, as diferenças entre os grupos foram identificadas por meio do teste qui-quadrado (comorbidades foi prevalência da terapia medicamentosa) ou teste t de Student não pareado (variáveis contínuas). Os efeitos das intervenções foram avaliados pela ANOVA mista de dois fatores (Statsoft, Statistic for Windows 4.3, Oklahoma, EUA), tendo como fatores grupo (GC e GTC) e fase do estudo (início e 12 semanas). Teste post-hoc de Newman-Keuls foi usado quando necessário. O valor de $p<0,05$ foi considerado significante e os dados foram apresentados em média \pm DP.

\section{Resultados}

O fluxograma de pacientes está representado na Figura 2. Oitenta e quatro pacientes foram triados, mas 35 foram excluídos por não atenderem aos critérios de elegibilidade $(n=7)$ ou não aceitaram participar ( $\mathrm{n}=28$, falta de disponibilidade para realização dos treinamento). Os 49 pacientes restantes foram alocados aleatoriamente no GC $(n=24)$ e no GTC $(n=25)$. Quatorze pacientes desistiram por circunstâncias não relacionadas ao estudo, de forma que a amostra final foi composta por 35 pacientes (GC, $\mathrm{n}=16$; GTC, $\mathrm{n}=19$ ).

Esses grupos tinham características iniciais semelhantes quanto à idade, grau de obesidade, níveis clínicos de PA, limitações da doença, comorbidades e uso de medicamentos (Tabela 1).

Os níveis de PA ambulatorial foram semelhantes entre os grupos no início do estudo, e nenhum deles apresentou qualquer mudança significante na PA de 24 horas, vigília e sono após as 12 semanas de intervenção (Tabela 2). 


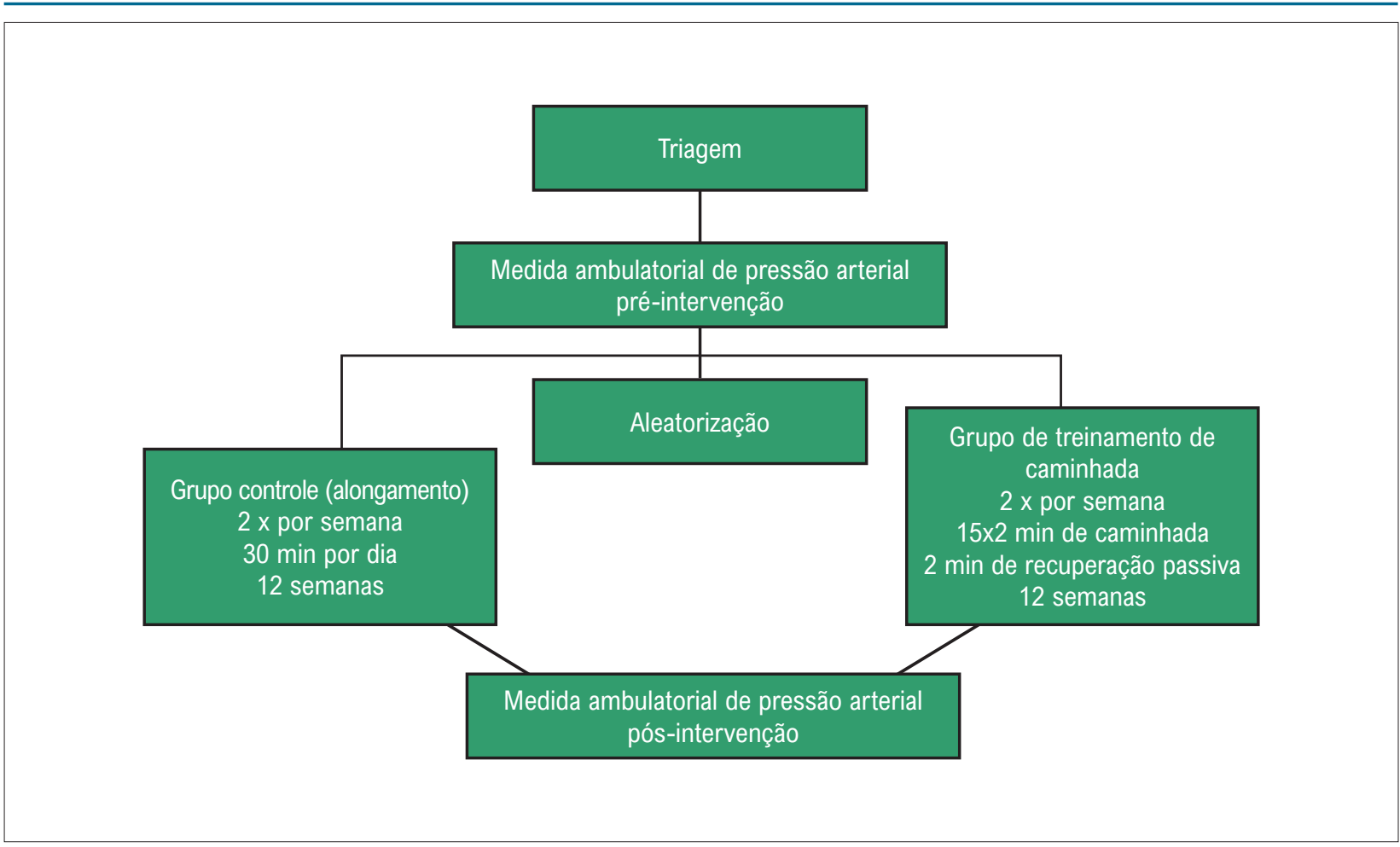

Figura 1 - Desenho experimental do estudo.

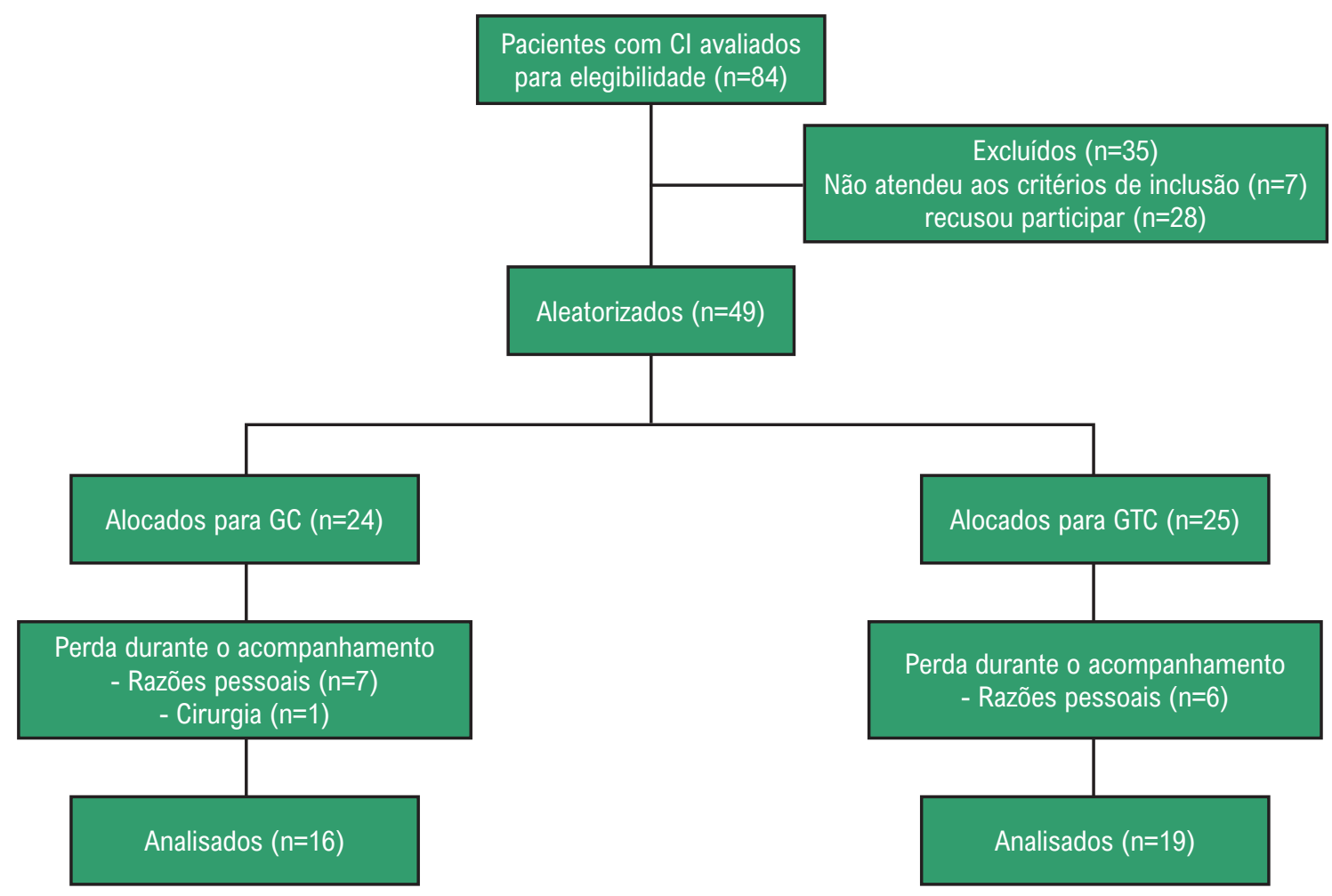

Figura 2 - Fluxograma do estudo Cl: claudicação intermitente, GC: Grupo controle, GTC: Grupo treinamento de caminhada 
Tabela 1 - Características dos pacientes alocados nos grupos controle e treinamento de caminhada

\begin{tabular}{|c|c|c|c|}
\hline & $G C(n=16)$ & GTC $(n=19)$ & Valor $p$ \\
\hline Idade (anos) & $62 \pm 7$ & $63 \pm 7$ & 0,64 \\
\hline Índice de massa corporal $\left(\mathrm{kg} / \mathrm{m}^{2}\right)$ & $25,7 \pm 3,9$ & $26,1 \pm 3,1$ & 0,76 \\
\hline Índice tornozelo-braquial & $0,60 \pm 0,12$ & $0,62 \pm 0,14$ & 0,61 \\
\hline Distância de início de claudicação $(\mathrm{m})$ & $319 \pm 152$ & $277 \pm 164$ & 0,45 \\
\hline Distância total caminhada (m) & $759 \pm 305$ & $624 \pm 255$ & 0,16 \\
\hline PA sistólica clínica (mmHg) & $136 \pm 19$ & $133 \pm 14$ & 0,60 \\
\hline PA diastólica clínica $(\mathrm{mmHg})$ & $79 \pm 10$ & $77 \pm 9$ & 0,53 \\
\hline \multicolumn{4}{|l|}{ Comorbidades } \\
\hline Obesidade (\%) & 12,5 & 10,5 & 0,55 \\
\hline Hipertensão (\%) & 81,3 & 84,2 & 0,89 \\
\hline Diabetes Mellitus (\%) & 25,0 & 21,1 & 0,61 \\
\hline Dislipidemia (\%) & 100,0 & 89,5 & 0,17 \\
\hline Fumantes atuais $(\%)$ & 37,5 & 26,3 & 0,38 \\
\hline Doença cardíaca/acidente vascular cerebral (\%) & 18,8 & 21,1 & 0,80 \\
\hline \multicolumn{4}{|l|}{ Terapia medicamentosa } \\
\hline Aspirina (\%) & 93,8 & 100,0 & 0,28 \\
\hline Estatina (\%) & 62,5 & 78,9 & 0,83 \\
\hline Inibidor da enzima de conversão da angiotensina (\%) & 43,8 & 68,4 & 0,20 \\
\hline Diuréticos (\%) & 25,0 & 47,4 & 0,17 \\
\hline Bloqueador do canal de cálcio (\%) & 18,8 & & 0,86 \\
\hline Hipoglicemiante oral (\%) & 18,8 & & 0,69 \\
\hline \multicolumn{4}{|l|}{ Número de anti-hipertensivos } \\
\hline Monoterapia & 50,0 & & 0,76 \\
\hline
\end{tabular}

Dados apresentados em média $\pm D P$ ou porcentagem (\%). PA: pressão arterial. Variável contínua - teste $t$ de Student não pareado. Variável categórica - teste do qui-quadrado. CG - Grupo controle, GTC - Grupo treinamento de caminhada.

Tabela 2 - Níveis de pressão arterial ambulatorial medidos no início e após o período de intervenção de 12 semanas no grupo treinamento de caminhada e o grupo controle

\begin{tabular}{|c|c|c|c|c|c|c|c|}
\hline & \multicolumn{2}{|c|}{$G C(n=16)$} & \multicolumn{2}{|c|}{ GTC $(n=19)$} & \multirow{2}{*}{$\underset{\text { grupo }}{P}$} & \multirow{2}{*}{$\begin{array}{c}P \\
\text { fase do estudo }\end{array}$} & \multirow{2}{*}{$\underset{\text { interação }}{\mathbf{P}}$} \\
\hline & início & 12 semanas & início & 12 semanas & & & \\
\hline \multicolumn{8}{|l|}{$24 \mathrm{~h}$} \\
\hline PA sistólica (mmHg) & $130 \pm 14$ & $132 \pm 15$ & $128 \pm 14$ & $126 \pm 11$ & 0,51 & 0,74 & 0,21 \\
\hline PA diastólica $(\mathrm{mmHg})$ & $78 \pm 7$ & $80 \pm 7$ & $78 \pm 12$ & $76 \pm 10$ & 0,44 & 0,42 & 0,16 \\
\hline PA média (mmHg) & $96 \pm 9$ & $98 \pm 8$ & $94 \pm 9$ & $93 \pm 9$ & 0,32 & 0,60 & 0,14 \\
\hline \multicolumn{8}{|l|}{ Vigília } \\
\hline PA sistólica (mmHg) & $135 \pm 14$ & $137 \pm 16$ & $130 \pm 14$ & $129 \pm 12$ & 0,16 & 0,74 & 0,44 \\
\hline PA diastólica $(\mathrm{mmHg})$ & $83 \pm 7$ & $84 \pm 7$ & $80 \pm 12$ & $79 \pm 11$ & 0,16 & 0,41 & 0,35 \\
\hline PA média (mmHg) & $101 \pm 9$ & $103 \pm 9$ & $96 \pm 10$ & $95 \pm 10$ & 0,08 & 0,60 & 0,25 \\
\hline \multicolumn{8}{|l|}{ Sono } \\
\hline PA sistólica (mmHg) & $119 \pm 16$ & $121 \pm 16$ & $124 \pm 16$ & $122 \pm 12$ & 0,50 & 0,85 & 0,51 \\
\hline PA diastólica $(\mathrm{mmHg})$ & $69 \pm 9$ & $71 \pm 8$ & $73 \pm 9$ & $71 \pm 11$ & 0,61 & 0,80 & 0,32 \\
\hline PA média $(\mathrm{mmHg})$ & $87 \pm 11$ & $89 \pm 11$ & $89 \pm 9$ & $89 \pm 9$ & 0,63 & 0,82 & 0,33 \\
\hline
\end{tabular}

Dados apresentados em média \pm desvio padrão. PA: pressão arterial. ANOVA de dois fatores (grupo e fase do estudo). CG: Grupo controle, GTC: Grupo treinamento de caminhada. 
Os índices de variabilidade da PA avaliados no início do estudo foram semelhantes entre o GTC e o GC. Houve interação significante entre grupos e a fase de estudo para os índices de variabilidade da PA sistólica e média (todos $\mathrm{P}<0,05)$, mostrando redução do $\mathrm{DP}_{24}, \mathrm{DP}_{\mathrm{vs}}$ e $\mathrm{VRM}_{24}$ da PA sistólica e média apenas no GTC (Tabela 3, Figura 3). Nenhum grupo teve qualquer mudança significante nos índices de variabilidade da PA diastólica.

\section{Discussão}

O principal achado deste estudo foi que 12 semanas de TC diminuíram os índices de variabilidade da PA sistólica e média, sem alterar os níveis de PA ambulatorial.

No presente estudo, 12 semanas de TC não alteraram a PA ambulatorial em pacientes com DAP, o que contrasta com estudos realizados com indivíduos normotensos e hipertensos ${ }^{19}$ que relataram consistentemente reduções em torno de $3 \mathrm{mmHg}$ para PA sistólica e diastólica ambulatorial após treinamento aeróbico. No entanto, 12 semanas de treinamento resistido também não alteraram a PA ambulatorial em pacientes com DAP. ${ }^{9}$ Assim, temse a hipótese de que episódios frequentes de isquemia durante as atividades diárias dos pacientes com DAP produzem dor de claudicação, estresse oxidativo e acúmulo metabólico, aumentando a atividade do nervo simpático e, consequentemente, mitigando qualquer possível efeito do exercício físico sobre os níveis de PA ambulatorial. ${ }^{20}$ Outra possível explicação, entretanto, pode ser a duração muito curta do programa, uma vez que um estudo anterior ${ }^{21}$ realizado com hipertensos idosos não mostrou alteração dos níveis de PA ambulatoriais após 6 meses de treinamento, após 12 meses de intervenção.
Apesar da ausência de alteração nos níveis de PA ambulatorial, foram observadas reduções nas variabilidades sistólica e média da PA ambulatorial para todos os índices de variabilidade: $\mathrm{DP}_{24}$, DPvs e VRM ${ }_{24}$. Esses resultados estão de acordo com estudo anterior com treinamento resistido em pacientes sintomáticos com DAP. ${ }^{9}$ Esse resultado é coerente com a ideia de que mudanças no controle autonômico precedem alterações nos níveis da PA, uma vez que a variabilidade da PA reflete principalmente o controle autonômico da PA. ${ }^{22,23}$ Além disso, esses resultados também estão de acordo com nossos achados anteriores de uma melhora na modulação autonômica cardíaca e sensibilidade barorreflexa, marcadores de controle autonômico, após TC em pacientes com DAP. ${ }^{7}$ A ausência de alterações na variabilidade da PA ambulatorial diastólica também é coerente com a ausência de efeitos do treinamento de caminhada na resistência vascular da panturrilha, como descrito anteriormente. ${ }^{7}$

Mesmo sem quaisquer alterações nos níveis de PA ambulatorial, a diminuição da variabilidade da PA ambulatorial obtida com o TC pode ter implicações clínicas relevantes. A variabilidade da PA tem sido associada à presença e progressão de lesões de órgãos alvo, bem como à incidência de eventos cardiovasculares, ${ }^{10}$ levando a um pior prognóstico cardiovascular. ${ }^{8}$ Assim, a diminuição induzida pelo TC pode ter impacto favorável no risco cardiovascular de pacientes com DAP, reforçando a recomendação do TC para esses pacientes.

Este estudo apresenta algumas limitações que devem ser reconhecidas. Foi realizado apenas com homens e as adaptações induzidas pelo treinamento podem diferir entre os sexos. ${ }^{24,25}$ Assim, estudos futuros devem investigar o impacto do TC na PA ambulatorial e sua variabilidade

Tabela 3 - Índices de variabilidade da pressão arterial ambulatorial avaliados no início e após o período de intervenção de 12 semanas para os grupos treinamento de caminhada e controle

\begin{tabular}{|c|c|c|c|c|c|c|c|}
\hline & \multicolumn{2}{|c|}{$G C(n=16)$} & \multicolumn{2}{|c|}{ GTC $(n=19)$} & \multirow{2}{*}{$\begin{array}{c}\text { P } \\
\text { grupo }\end{array}$} & \multirow{2}{*}{$\begin{array}{c}P \\
\text { fase do } \\
\text { estudo }\end{array}$} & \multirow{2}{*}{$\underset{\text { interação }}{\mathbf{P}}$} \\
\hline & início & 12 semanas & início & 12 semanas & & & \\
\hline \multicolumn{8}{|l|}{$\mathrm{DP}_{24}$} \\
\hline PA sistólica (mmHg) & $14,6 \pm 3,0$ & $15,5 \pm 3,9$ & $13,3 \pm 2,8$ & $11,8 \pm 2,3^{*} \#$ & 0,01 & 0,65 & 0,04 \\
\hline PA diastólica $(\mathrm{mmHg})$ & $10,9 \pm 1,8$ & $11,2 \pm 1,7$ & $9,7 \pm 2,3$ & $10,0 \pm 2,5$ & 0,06 & 0,49 & 0,68 \\
\hline PA média $(\mathrm{mmHg})$ & $12,0 \pm 2,6$ & $13,0 \pm 3,0$ & $11,0 \pm 1,7$ & $10,4 \pm 1,9 \#$ & 0,01 & 0,71 & 0,04 \\
\hline \multicolumn{8}{|l|}{ DPvs } \\
\hline PA sistólica (mmHg) & $12,2 \pm 2,4$ & $12,7 \pm 3,0$ & $12,1 \pm 2,4$ & $10,7 \pm 2,5^{\star} \#$ & 0,18 & 0,27 & 0,03 \\
\hline PA diastólica (mmHg) & $8,7 \pm 1,3$ & $9,0 \pm 1,6$ & $9,0 \pm 1,8$ & $8,9 \pm 2,2$ & 0,98 & 0,95 & 0,48 \\
\hline PA média $(\mathrm{mmHg})$ & $10,0 \pm 2,1$ & $10,7 \pm 2,2$ & $10,1 \pm 1,6$ & $9,1 \pm 1,7^{\star} \#$ & 0,23 & 0,82 & 0,01 \\
\hline \multicolumn{8}{|l|}{ VRM $_{24}$} \\
\hline PA sistólica (mmHg) & $9,4 \pm 2,1$ & $10,7 \pm 2,4^{*}$ & $9,4 \pm 2,3$ & $8,8 \pm 2,2 \#$ & 0,18 & 0,28 & 0,02 \\
\hline PA diastólica (mmHg) & $6,9 \pm 1,8$ & $7,3 \pm 1,8$ & $7,3 \pm 2,3$ & $7,2 \pm 1,6$ & 0,75 & 0,67 & 0,54 \\
\hline PA média $(\mathrm{mmHg})$ & $8,1 \pm 1,9$ & $8,6 \pm 1,7$ & $8,0 \pm 1,7$ & $7,2 \pm 1,5^{\star} \#$ & 0,15 & 0,88 & 0,01 \\
\hline
\end{tabular}

Valores apresentados em média \pm desvio-padrão. $D P_{24}=$ desvio-padrão ponderado de 24 horas; $D P v s=$ desvio-padrão ponderado em vigilia e durante o sono; VRM = variabilidade real média. ANOVA de dois fatores (grupo e fase do estudo). *Diferente do início $(p<0,05)$; \# Diferente do GC $(p<0,05)$. GC: Grupo controle, GTC: Grupo treinamento de caminhada, PA: pressão arterial. 


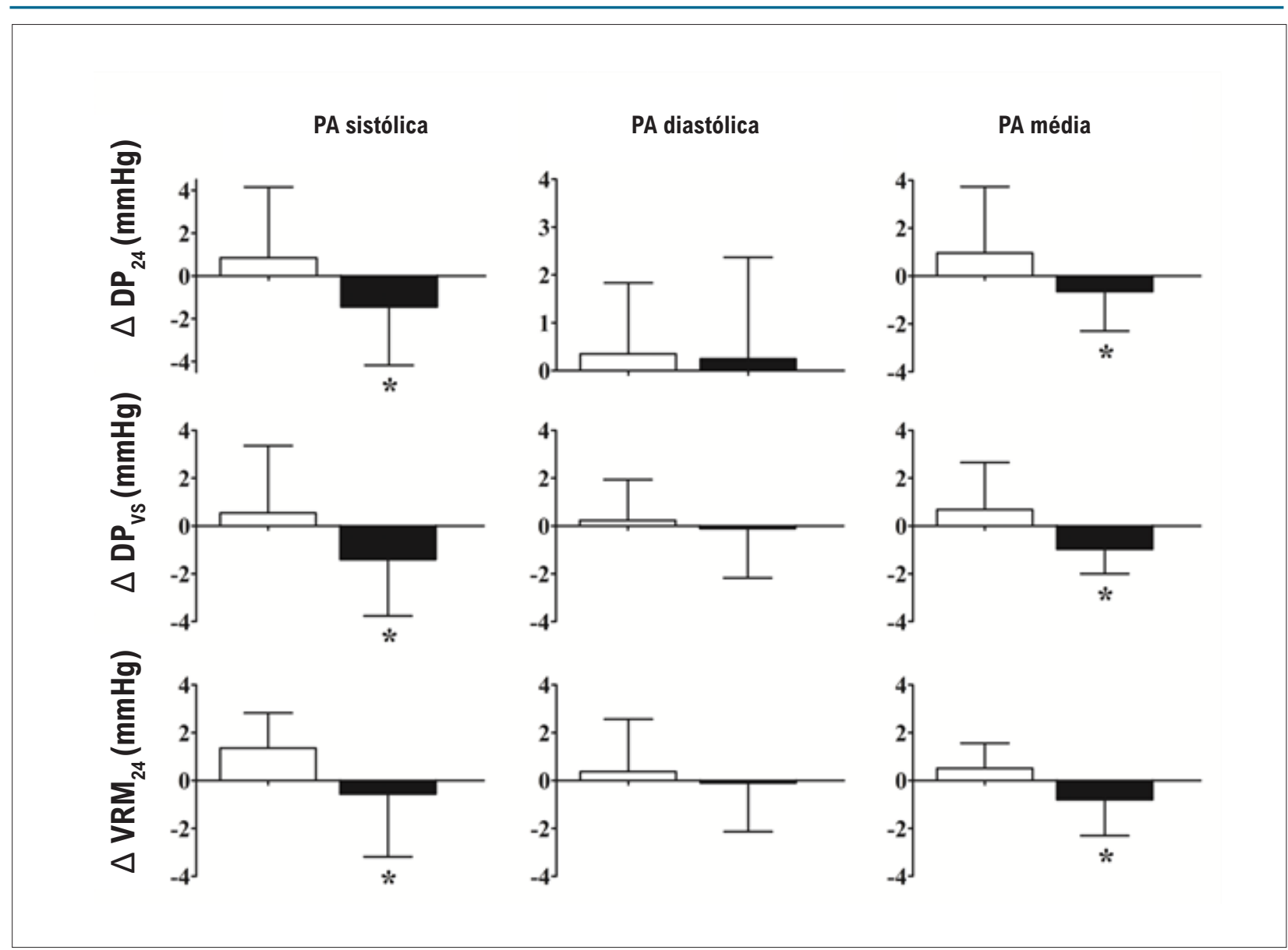

Figura 3 - Alteração absoluta $(\Delta)$ da variabilidade da pressão arterial ambulatorial para o grupo controle (GC - barras brancas) e grupo de treinamento de caminhada (GTC - barras pretas). PA: pressão arterial; $D P_{24}$, desvio-padrão acima de 24 horas ponderado pelo intervalo de tempo entre leituras consecutivas; DPvs, a média dos DPs diurnos e noturnos ponderados para a duração do intervalo diurno e noturno, VRM ${ }_{24}$ variabilidade real média ponderada para o intervalo de tempo entre leituras consecutivas de registros de PA ambulatorial de 24 horas. ${ }^{*} p<0,05$ vs. GC.

também em mulheres, principalmente idosas, que podem apresentar maior risco cardiovascular do que os homens. ${ }^{24}$ O presente estudo também examinou apenas pacientes com sintomas de claudicação, e novos estudos devem examinar os efeitos do TC em outros grupos de pacientes, como os assintomáticos (estágio 1) e naqueles que apresentam diminuição dos níveis pressóricos ambulatoriais após o TC. Por fim, o programa durou 12 semanas, duração que melhora a capacidade funcional e os parâmetros clínicos cardiovasculares desses pacientes, ${ }^{7}$ mas um período de treinamento mais longo pode ser necessário para diminuir os níveis pressóricos ambulatoriais.

\section{Conclusão}

Em conclusão, 12 semanas de TC diminui a variabilidade ambulatorial da PA em homens com DAP sintomática.

\section{Contribuição dos autores}

Concepção e desenho da pesquisa: Chehuen M, Cucato GG, Zerati AE, Leicht A, Ritti-Dias RM, Forjaz CLM;
Obtenção de dados: Chehuen M, Cucato GG; Análise e interpretação dos dados: Chehuen M, Cucato GG, Forjaz CLM; Análise estatística e Obtenção de financiamento: Forjaz CLM; Redação do manuscrito: Chehuen M, Cucato GG, Carvalho C, Wolosker N, Ritti-Dias RM; Revisão crítica do manuscrito quanto ao conteúdo intelectual importante: Chehuen M, Cucato GG, Carvalho C, Zerati AE, Leicht A, Wolosker N, Ritti-Dias RM, Forjaz CLM.

\section{Potencial conflito de interesse}

Não há conflito com o presente artigo

\section{Fontes de financiamento}

O presente estudo foi financiado pelo CNPQ (442507/2014-3; 304436/2018-6), FAPESP (2015/138000) e CAPES (0001).

\section{Vinculação acadêmica}

Este artigo é parte de tese de doutorado de Marcel Chehuen pela Universidade de São Paulo. 


\section{Referências}

1. Gerage AM, Correia MA, Oliveira PML, Palmeira AC, Domingues WJR, Zeratti AE, et al. Physical Activity Levels in Peripheral Artery Disease Patients. Arq Bras Cardiol. 2019;113(3):410-6.

2. Wu A, Coresh J, Selvin E, Tanaka H, Heiss G, Hirsch AT, et al. Lower Extremity Peripheral Artery Disease and Quality of Life Among Older Individuals in the Community. J Am Heart Assoc. 2017;6(1):e004519.

3. Ritti-Dias RM, Correia MA, Andrade-Lima A, Cucato GG. Exercise as a therapeutic approach to improve blood pressure in patients with peripheral arterial disease: current literature and future directions. Expert Rev Cardiovasc Ther. 2019;17(1):65-73.

4. Bhatt DL, Steg PG, Ohman EM, Hirsch AT, Ikeda Y, Mas JL, et al. International prevalence, recognition, and treatment of cardiovascular risk factors in outpatients with atherothrombosis. JAMA. $2006 ; 295(2): 180-9$.

5. Svensson P, de Faire U, Niklasson U, Ostergren J. Office blood pressure underestimates ambulatory blood pressure in peripheral arterial disease in comparison to healthy controls. J Hum Hypertens. 2004;18(3):193-200.

6. Lima A, Chehuen M, Cucato GG, Soares AHG, Askew CD, Barbosa J, et al. Relationship between walking capacity and ambulatory blood pressure in patients with intermittent claudication. Blood Press Monit. 2017;22(3):115-21.

7. Chehuen M, Cucato GG, Carvalho CRF, Ritti-Dias RM, Wolosker N, Leicht AS, et al. Walking training at the heart rate of pain threshold improves cardiovascular function and autonomic regulation in intermittent claudication: A randomized controlled trial. J Sci Med Sport. 2017;20(10):886-92.

8. Yang WY, Melgarejo JD, Thijs L, Zhang ZY, Boggia J, Wei FF, et al. Association of Office and Ambulatory Blood Pressure With Mortality and Cardiovascular Outcomes. JAMA. 2019;322(5):409-20.

9. Gomes APF, Correia MA, Soares AHG, Cucato GG, Lima A, Cavalcante $\mathrm{BR}$, et al. Effects of Resistance Training on Cardiovascular Function in Patients With Peripheral Artery Disease: A Randomized Controlled Trial. J Strength Cond Res. 2018;32(4):1072-80.

10. Mena LJ, Felix VG, Melgarejo JD, Maestre GE. 24-Hour Blood Pressure Variability Assessed by Average Real Variability: A Systematic Review and Meta-Analysis. J Am Heart Assoc. 2017;6(10):e006895.

11. Cardoso CG, Jr., Gomides RS, Queiroz AC, Pinto LG, da Silveira Lobo F, Tinucci T, et al. Acute and chronic effects of aerobic and resistance exercise on ambulatory blood pressure. Clinics (Sao Paulo). 2010;65(3):317-25.

12. Gerhard-Herman MD, Gornik HL, Barrett C, Barshes NR, Corriere MA, Drachman DE, et al. 2016 AHA/ACC Guideline on the Management of Patients With Lower Extremity Peripheral Artery Disease: A Report of the American College of Cardiology/American Heart Association Task Force on Clinical Practice Guidelines. J Am Coll Cardiol. 2017;69(11):e71-e126.

13. Hirsch AT, Haskal ZJ, Hertzer NR, Bakal CW, Creager MA, Halperin JL, et al. ACC/AHA 2005 Practice Guidelines for the management of patients with peripheral arterial disease (lower extremity, renal, mesenteric, and abdominal aortic): a collaborative report from the American Association for Vascular Surgery/Society for Vascular Surgery, Society for Cardiovascular Angiography and Interventions, Society for Vascular Medicine and Biology, Society of Interventional Radiology, and the ACC/ AHA Task Force on Practice Guidelines (Writing Committee to Develop Guidelines for the Management of Patients With Peripheral Arterial Disease): endorsed by the American Association of Cardiovascular and Pulmonary Rehabilitation; National Heart, Lung, and Blood Institute; Society for Vascular Nursing; TransAtlantic Inter-Society Consensus; and Vascular Disease Foundation. Circulation. 2006;113(11):e463-654.

14. Boulton AJ, VinikAI, Arezzo JC, Bril V, Feldman EL, Freeman R, etal. Diabetic neuropathies: a statement by the American Diabetes Association. Diabetes Care. 2005;28(4):956-62.

15. Guirguis-Blake JM, Evans CV, Redmond N, Lin JS. Screening for Peripheral Artery Disease Using the Ankle-Brachial Index: Updated Evidence Report and Systematic Review for the US Preventive Services Task Force. JAMA. 2018;320(2):184-96.

16. Gardner AW, Skinner JS, Cantwell BW, Smith LK. Progressive vs singlestage treadmill tests for evaluation of claudication. Med Sci Sports Exerc. 1991;23(4):402-8.

17. Hansen TW, Thijs L, Li Y, Boggia J, Kikuya M, Björklund-Bodegård K, et al. Prognostic value of reading-to-reading blood pressure variability over 24 hours in 8938 subjects from 11 populations. Hypertension. 2010;55(4):1049-57.

18. Cucato GG, Chehuen Mda R, Costa LA, Ritti-Dias RM, Wolosker N, Saxton $J M$, et al. Exercise prescription using the heart of claudication pain onset in patients with intermittent claudication. Clinics (Sao Paulo). 2013;68(7):974-8.

19. Cornelissen VA, Buys R, Smart NA. Endurance exercise beneficially affects ambulatory blood pressure: a systematic review and meta-analysis. J Hypertens. 2013;31(4):639-48.

20. Muller MD, Drew RC, Blaha CA, Mast JL, Cui J, Reed AB, et al. Oxidative stress contributes to the augmented exercise pressor reflex in peripheral arterial disease patients. J Physiol. 2012;590(23):6237-46.

21. Seals DR, Reiling MJ. Effect of regular exercise on 24-hour arterial pressure in older hypertensive humans. Hypertension. 1991;18(5):583-92.

22. Chaar LJ, Alves TP, Batista Junior AM, Michelini LC. Early Training-Induced Reduction of Angiotensinogen in Autonomic Areas-The Main Effect of Exercise on Brain Renin-Angiotensin System in Hypertensive Rats. PLoS One. $2015 ; 10(9): \mathrm{e} 0137395$.

23. Masson GS, Costa TS, Yshii L, Fernandes DC, Soares PP, Laurindo FR, et al. Time-dependent effects of training on cardiovascular control in spontaneously hypertensive rats: role for brain oxidative stress and inflammation and baroreflex sensitivity. PLoS One. 2014;9(5):e94927.

24. Correia MA, de Sousa ASA, Andrade-Lima A, Germano-Soares AH, Zerati $A E$, Puech-Leao $P$, et al. Functional and Cardiovascular Measurements in Patients With Peripheral Artery Disease: comparison between men and women. J Cardiopulm Rehabil Prev. 2020;40(1):24-8.

25. Cucato GG, Ritti-Dias RM, Franco FG, de Mattos LD, Cendoroglo MS, Wolosker N, et al. Influence of peripheral arterial disease on daily living activities in elderly women. J Vasc Nurs. 2016;34(2):39-43. 